\title{
Can Mental Modularity Find Any Substitute?
}

\author{
CUI Yanying \\ School of Foreign Languages, Taiyuan University of Science and Technology, China \\ Received: July 22, 2021 \\ Accepted: November 16, 2021 \\ Published: January 31, 2022
}

This paper is funded by "Research on the Interaction and Sociality of Mind-brain Module"(2019041019-2), Shanxi Soft Science Research Project.

To cite this article: CUI Yanying. (2021). Can Mental Modularity Find Any Substitute?. Asia-Pacific Journal of Humanities and Social Sciences, 1(4), 063-071, DOI: 10.53789/j. 1653-0465.2021.0104.009.p

To link to this article: https://doi. org/10.53789/j. 1653-0465.2021.0104.009.p

\begin{abstract}
Mental modularity claims particular importance in the study of the philosophy of language. Innatists think language, similar to other cognitive modules, is a separate module, which performs the particular functions and processes corresponding input and is not affected by other modules. The fact is that different modules interact with each other and affect each other. Language instinct does not hold. Hence mental modularity goes broke. Ultimately, a feasible approach, the complex system theory, is needed to understand language faculty and interpret "the black box".
\end{abstract}

Keywords: complex system theory; mental modularity; grammar gene; language instinct

Notes on the contributor: CUI Yanying is a lecturer at the School of Foreign Languages, Taiyuan University of Science and Technology. Her research interest lies in theoretical linguistics and the philosophy of language. Her email address is cyy19770522@126.com.

\section{Introduction}

Western philosophical tradition regards the mind as an ability. Cognitive science enables computers to simulate the human mind. A computerized human brain model appears to be capable of complex reasoning, exactly the same way human beings cognize the world. From a mind-computing perspective, it seems possible to associate a particular function with a particular part of a computer. Mental modules function through the neuron system in which a module handles its specific information, without other modules involved, just as the computer components are relatively independent, interrelated and modularized. If one module is damaged, the affected feature does not function properly, but other modules will usually work. Since all modules process their specified information, the corresponding module cannot be activated until specific information appears and needs to be processed. When a module is activated, the module-specific process gets running. What about the language module? Does this prove language is a mental module, or does language instinct presupposes there is such a language 
module? The relationship between language and cognition is extremely complex. In order to make it clear, we also need to deal with the relationship between language and other cognitive aspects, which requires us to reexamine the grammar structure.

\section{Is There Any "Language Module"?}

Pinker believes that language is an instinct, a module of the brain, a recognizable part of the brain, and even certain sequences of genes locate the language module somewhere in the brain. If the nerve is damaged, the language module is affected, but the other modules stay untouched. If other brain modules get damaged, the language module stays intact, which arises a "language gene". "There are many cases in which the brain is damaged, the nerves are damaged, the language faculty is damaged, but other cognitive abilities are intact" (Pinker 1994). This also seems to indicate that language is an independent module. Different modules may be spatially discrete and located in specific parts of the brain, which is true of the language module. Three things are needed to be an independent module. One is that there is a language-related module in the brain. The second is the dissociation of language module from other cognitive abilities, including knowledge of other aspects of languages, such as vocabulary development and grammar acquisition. If language faculty is impaired, other cognitive functions are left unaffected since the knowledge modules are independent. The third point is a clear reference point indicating that language develops at a particular period and is unaffected by another period.

The language module is said to be the result of lateralization in the brain, which contributes to the left and right hemispheres for complex organisms. The lateralization is by no means symmetrical. Both sides perform the functions related to language. Generally, the left side is used to process grammar acquisition and vocabulary development, and the right side functions to process what is heard. French neurologist Paul Broca first discovered that people who suffered from damage in the left frontal lobe of the brain could not produce any speech but were able to comprehend language; later, Carl Wernicke, a German, described the area that seemed to be significant to language processing. Broca's patients could understand the language but were severely impaired in the vocal areas and unable to speak. On the other hand, Wernick's patients were able to speak, but could not understand the language. Whether one can't speak or understand is aphasia in one way or another. Broca's patient is of expressive aphasia, and the patient suffers some damage to his language area. If the damage is not severe, a person with Broca's aphasia can slowly and painfully produce words, but with poor grammar and discontinuous speech (Caplan 2006). In contrast, Wernicke's encephalopathies are of receptive aphasia, and patients with this aphasia can hear voices and read the print but cannot understand language.

Broca's area is indeed involved in the production of language. Can it be explained that Broca's area is of the language module? Pinker acknowledges that "no one has yet located a language organ or a grammar gene, but the search is still going on” (Pinker 1994). At least Broca's area and Wernicke's area may be related to language genes. Other cognitive abilities remain unaffected while either illness or a sudden shot impairs speech. This shows that language is independent of other cognitive abilities, which provides a basis for the theory of language modules

Cognitive scientist Howard Gardner also pointed out that language skills were impaired, and other mental abilities such as drawing, calculating, recognising maps, setting alarms, and executing commands were unaffect- 
ed (Gardner 1976). What is the basis of the "grammar gene"? London family is a case in point. Once there was a study about a London family, half of whom had trouble learning languages. About 15 members had problems with speech sounds, morphology, and syntax for three generations. The experiments also showed that these members appeared to have a genetic variant that affected a gene FOXP2 (Lai 2001). Nativists think that language is genetically determined and located somewhere in the brain like a language module. If the module wants to be an independent one, it needs to meet the so-called double dissociation: Just as Broca's patients could not speak but could understand language, Wernicke's patients could never understand the language but were able to produce fluent speech. The same is true of other cognitive abilities. Damage to language module makes no difference to other cognitive modules working, and vice versa.

Pinker's "chatterboxes" illustrate the separation of verbal ability from other cognitive abilities. Patients with this syndrome have difficulty learning to read and write but can still have an understanding of language due to their chromosome 11 defects caused by severe mental damage. Their intelligence quotient score is low, but their language ability is extraordinary. However, they cannot tie their shoelaces. They cannot tell right from left. The other type of patients suffers from dyslexia, a special language disorder, with normal intelligence. The symptoms of dyslexia are different from those of Broca's aphasia. Patients with dyslexia are also the result of genetic abnormalities.

Either "chatterbox" or "dysphasia" point to the double dissociation between language and intelligence. Either language module is impaired with intelligence unaffected; or the language is intact with poor intelligence, which seems to confirm the theory of language module, a theory of language innateness, and Chomsky calls it "universal grammar", that is, the development of language, or any other module, involves a genetic endowment that converts linguistic data into the linguistic experience and guides the general development of language (Chomsky 2011). For language, we can look upon genetic endowment as a factor specific to human beings.

Chomsky argued that language is a separate module. Language data are all the words that children encounter first at the very early stage. They are cooing and babbling. According to language instinct, the maturity of language module, the maturity of universal grammar, originates in telegraphic vocabulary and finally develops into a mature grammar, which links words into sentences according to grammatical categories and syntactic rules. This is also an assumption that the language acquisition device is activated to allow syntax to emerge at a certain period. Locke once proposed that "grammatical mechanisms" begin to work at around two and proliferate between two or three years old. Grammar needs to include some basic vocabulary before it emerges. It is hard to learn vocabulary at the babbling stage, but once the language module gets activated and grammar appears, learning vocabulary will be natural and fast because "language is a module and it is not that much related to language development" (Smith 1999). Therefore, the principle of universal grammar promotes the rapid development of grammar.

\section{How is Language Module Possible?}

Is there any "chatterbox"? Are there any grammar genes? Is there any language acquisition mechanism that promotes the development of Universal Grammar?

First, let us look at language regions: Is there really a part of the brain where language is located? Broca's 
area has been associated with language production for over a hundred years, but can we say that this area is just the place where a language is located, a location responsible for language production, and a location that rules out other possibilities? Broca's area also seems to work for important aspects of the nonverbal task, such as controlling voluntary movement, even recognizing discordant tunes in music (Maess et al. 2001). Moreover, the damage in Broca's area does not mean that there is no room for improvement after the complete damage of language (Plaza et al. 2009). For example, one computer engineer suffered from chronic tumors, which was later removed from the brain and which, as a result, destroyed Broca's area, causing small areas of language damage, allowing patients to communicate normally after three months of brain recovery (Corballis 2003). Broca's area is not only responsible for language output, but also related to other cognitive activities. Broca's area allows us to perceive the behavior of others and to respond to specific behaviors, especially to body language and gestures (Pulvermilllers 1999). Therefore, language may be developed from gestures, and the relationship between Broca's area and language output may be the result of processing and recognizing gestures. Just as Zerilli put it, neighbouring modules have similar response properties: Laminar and columnar changes are for the most part smooth - not abrupt - as one moves across the cortex, and adjacent modules do not differ markedly from one another in their basic structure and computations (if they even differ at all when taken in such proximity) (Zerilli 2019). It is an exact echo to Junge and Dennett when they have said that brain areas contain a large amount of structural redundancy, that is, many neurons or collections of neurons that can potentially perform the same class of functions, instead of a single neuron or small neural tract playing roles in many high-level processes. Possibly distinct subsets of neurons within a specialized area are born with similar competencies and, hence, are redundant and available to be assigned individually to specific uses. (Junge and Dennett 2010).

Moreover, there is no consensus on the exact location of Broca's area (Poeppel 1996), which seems to be everywhere, possibly in any part of the brain (Pulvermüller 1999; Dqbrowska 2004).

Now let's talk about the grammar gene FOXP2. First, grammar genes are not specified for language. They also affect facial and oral muscles, which may not have anything to do with language. Moreover, the FOXP2 gene is not unique to humans and is also found in other mammals. It is genetically similar to other primate brains (Enard et al. 2002). The gene also plays an important part in heart, gut and lung development. Thus, "human FOXP2 gene does contribute to individual language development, but it is by no means a grammatically specific blueprint" (Dqbrowska 2004).

The dissociation of language from other cognitive abilities is equally indefensible. There is no double dissociation at all. Pinker's chatterboxes exhibit "extraordinary verbal abilities", but this is only the result of being compared with their own visual, spatial and reasoning abilities. We cannot conclude that they are linguistic geniuses (Bates \& Goodman 1999). "Chatterboxes" are of lower intelligence quotients than children of the same age. Their phonetic memory is unparalleled, but their ability to remember new words is not so good as that of average children, and their ability to understand words and sentences and generate sentences is also lower than that of normal children. Although they eventually succeed in language acquisition, both their production and comprehension lag behind their normal peers (Harris et al. 1997). They can indeed acquire difficult vocabulary and master complex grammatical structures (Bellugi et al. 1994). This is unusual for people with low IQs. Their learning strategies were also lower than normal children. They refer to "new words as parts of objects, while other children refer to new words as whole objects (Karmiloff-Smith 2001). They cannot know whether a sentence 
is grammatical or not and cannot use complex grammar to make a sentence. The so-called "chattering symptom" is exactly the result of the defect of language behavior and of abnormal language development. In short, it is neither accurate nor scientific to say that a "chatterbox" is by any means a linguistic genius.

Patients with special language disorder have delayed language behaviors and have delayed non-verbal behaviors such as imagination ability, the ability to represent objects with symbols, and the ability to shift their attention from one thing to another (Townsend et al. 1995). As a result, they are impaired not only in language but also in other mental abilities. Their ability to form words is seriously affected and morphological learning, especially inflectional morphemes, seems really difficult to them. They cannot recognize inflections in the sentences, even the added minute suffix or a simple sound unit. Therefore, patients whose language gets impaired have language problems and experience hearing problems, and their phonological development lags behind, making it difficult to internalize phonological knowledge (Mody et al. 1997).

Let's look at language acquisition. Children acquire vocabulary slowly at first, but the process accelerates after a year and a half, and then comes grammar acquisition. There is continuity between grammar and lexical development, and grammar behavior is closely related to vocabulary building. That is, the lexical quantity makes a difference to grammar acquisition (Bates et al. 1997). In this sense, grammar is not encapsulated knowledge. It is closely related to vocabulary development and can be predicted. Grammar is not a separate mental module, so language is not instinctive. In short, grammar and vocabulary acquisition takes on a continuum: with one end being vocabulary and the other grammar. Grammar emerges at a particular time during the development of a language.

\section{Does Mental Modularity Make Any Sense?}

The theory of mind modules has a long history, originating in the materialist tradition that mental functions are located in specific areas of the brain. It can be better explained on that tradition that the layout of the brain can be named regions that perform the corresponding functions. It is also the result of the mind-brain reduction. Therefore, the modular theory of mind endorses the one-to-one correspondence between mental functions and brain regions. The modular theory holds that both language faculty and other cognitive abilities are modules. As the spokesman of the instinctive view of language, Pinker thinks cognition is a set of instincts, like complex circuits, each of which solves a particular computational problem and performs a particular function. Pinker is an advocate of modularity, which he thinks can explain the workings of the mind (Pinker 1994). For him, the mind is a collection of special tools designed to perform a particular function, ranging from "choosing a partner, starting a family, choosing a healthy diet, making a decision and recognizing faces to reading mental states and understanding language" (Pinker 1997).

As far as modularity is concerned, all the explanations appear firm. However, module theory also faces difficulties and challenges. On the one hand, language is the best example to explain modular theory. On the other hand, language also makes modular theory difficult to stand, especially for a double dissociation. The nervous system is regional, a system that processes a particular kind of information, either verbal information or other kinds of information. Brain regions are interconnected, and cognitive abilities vary but are intertwined (Prinz $2006)$. 
The primary challenge of psychological modularization is logic. The puzzle was put forward by Fodor, a leading figure in the modular theory of mind. How does the mind know which module gets activated to process the entering information? The question also escalates to: How does the mind identify information of different types and specify corresponding modules? Does it also require a meta-module that knows everything upfront? Since modules only deal with certain types of information, is there any central system that connects modules and determines which module handles which type of information? There must be a central intelligence system that is focused on the mind in general. But this is beyond Fodor's modular view. He believes that modules encapsulate information. A module only deals with a particular kind of information and does not allow different modules to communicate with each other. In fact, it is far from true since countless examples show they are interacting with each other.

The McGurk effect could explain the interaction between brain modules. Scottish psychologist McGurk has found that in phonetic perception, the interaction between hearing and vision will affect the hearing and cause mishearing (McGurk 1976). If the sound we see doesn't match The McGurk hear, we perceive a third sound. This experiment shows that the visual and auditory systems interfere, suggesting that the mental module cannot be totally closed. The "contact illusion" can also explain the communication and interaction between modules (Hotting et al. 2004).

The brain link between sight and touch is another case in point. Researchers have shown that the part of the brain processing visual information is also indispensable to touch. This study, led by Emory University, confirms the role of the visual cortex in touch perception. These findings confirm the relevance of processing sensory information to such conditions as altered blindness, deafness or numbness, and ultimately improved communication methods for individuals with these diseases. These sensory interactions found in their study may be more common than we had generally assumed and even recognized, and the visual cortex is activated even in blind people when they read braille. According to this study, this is not at all a surprise (Preston 1999). Just as what the plasticity of senses views amputees experience phantom limbs because limb-detectors get rewired to neighboring cells (Chen et al. 2002). In this way, module activation seems to be input-oriented; hence, the modular view can hardly hold, which is true of the language module.

The modular view promises internalism. Pinker advocates a modular theory of the mind, which holds that there is an internal mechanism for developing stereoscopic vision. This inherent mechanism requires assumptions about unpredictable inputs (Pinker 2002). If we are rationalists, how do we explain the evolution of the mind? If a module is not closed or encapsulated, it doesn't have to occupy a particular part of the brain. Does that mean that humans are intelligent and capable of making decisions? Therefore, we can predict that the nervous system of modules will evolve at different speeds, which is different from other modules. In other words, the brain is a mosaic of different modules, evolving and developing according to different functions.

However, neuroscientist and philosopher Stephen Quartz used the results of mammalian brain evolution to conclude that mammalian brain research, including homo sapiens research, suggesting that the brain is coordinated and not Mosaic (Quartz 2002). Parts of the adult brain, such as the cerebellum, striatum, and cortex, develop in unison. If the evolution of the brain is what is called a mosaic, then the ratio of different parts of the brain, by comparison, takes on different forms, in different proportions. In fact, parts of the mammalian brain evolve in coordination with the size of the brain. Quartz believes that the coordinated development of the brain, rather 
than mosaic development, is the result of the interaction between the brain, body and the surrounding environment.

The above experiments show that the modular theory cannot stand up to the scientific test, and the viewpoints of the modular theory cannot be falsified and are never of scientific nature: It is impossible to classify various human behaviors into different modules in the context of human behavior and interaction. Therefore, mental abilities are not required to have specific areas to process specific information. Instead, they require the coordinated development of various parts.

\section{Complex System Theory: A Substitute for Modular Theory?}

Module evolution takes the form of value increment, neither the result of a pre-designed blueprint nor the environmental impact. Evolution occurs in due course, in relation to other changes, and co-evolves with the components. The relationship between the human brain and language is similar to the giraffe story: The survival of the fittest. The giraffe's neck is such that they can reach leaves, but their long necks go hand in hand with other changes, including cardiovascular changes in such a way that they enable blood to get access to the brain and its whole body. At the same time, this change also requires the development of other parts of the body, the hind legs shorter than the fore legs, in case the giraffe falls. In Gould's words, a long neck must be associated with modifications in nearly every part of the body - long legs to accentuate the effect, and a variety of supporting structures, with bones, muscles, and ligaments involved to hold up the neck (Gibbs et al. 2010). What's more, the giraffe's pharyngeal nerve passage does so, allowing it to travel from the brain through the aortic arch to the pharynx, which is merely an organ co-evolution, that is, all related parts must be changed in one fell swoop, and such coordinated modification is the result of internally generated variation (Gould 1998). Evolution is not a mechanical process.

So do languages. The quantitative change of coordinated development leads to the great qualitative change like the human brain. In addition to physiological changes that require oral production, humans also require changes of words and grammar in the memory system to adapt to the production of legitimate sentences. In the same way, a kind of meaning coding system is needed to match meaning with the phonetic symbols for the convenience of cognition. Indeed, human reading skills derive from both social experience and language training. Broca's area, now the language area, evolves from processing other functions earlier, recognising gestures first and foremost and later promoting language production. Changes in one region of the brain also entail changes in the rest of it. Co-evolution is an established development pattern, just as bees and flowers co-evolve to make the best from each other.

Complex system theory holds that any system can be regarded as a self-organizing and self-adaptive system. This way, it can be used to explain mental problems. Each factor involved is the internal reorganization of the operating system(Dorogovtsev et al. 2003). Just like the highway traffic, it is a good example to illustrate self-organization and self-coordination. Factors like traffic lights, intersections, ring roads, and other factors together affect and organize the traffic system. Other factors are equally important, drivers passing through the accident vehicles and looking around, causing traffic congestion behind and slowing down for observation. The same is true of mental modules. Psychologist Robert Gibbs believes that the brain can be thought of as a self-or- 
Asia-Pacific Journal of Humanities and Social Sciences

ganizing system: A complex adaptive system. We do not deny other types of evolutionary adaption. We have regular discrimination and resilience, and we self-regulate and respond to the nervous system, of which language and other cognitive abilities are just parts (Karmiloff-Smith et al. 1995).

Fodor and Chomsky agree that brain modules are not inborn but are shaped by divisions of labor (Smith 1994). Modular development is a gradual evolution, step by step, little by little. At first, the activation of information in the brain may be widespread over the brain. Over time, the certain line begins to process a certain type of information gradually. Modularization and specialization turn out to be effective for the brain to develop and manage massive information. As the brain processes information efficiently, specific circuits become specialized and modularized.

\section{Concluding Remarks}

The concept of a module, especially Fodor's classical module theory, is a controversial perspective at most. Why does the theory of language instinct suggest that the mind is modular? The human mind can see through nature's hidden problems, but does this mean we need modularity to reject our actual predicament? The unfalsifiability of Fodor's modular theory is a barrier to lead to science, which is not feasible at all. The interaction between language and other mental modules results from the coordinated development of the brain-mind system.

\section{References}

Bates, E., Thal, D., Trauner, D., Fenson, J., Aram, D., Eisele, J. \& Nass, R. (1997). From first words to grammar in children with focal brain injury. Developmental Neuropsychology, 13, 275-343.

Bates, E. \& Goodman, J. (1999). On the emergence of grammar from the lexicon. In B. MacWhinney (Ed.), The emergence of language. Mahwah, NJ: Lawrence Erlbaum.

Bellugi, U., Wang, P. \& Jernigan, T. L. (1994). Williams syndrome: an unusual neuropsychological profile. In Broman, S. and Grafman, J. (eds. ), Atypical cognitive deficits in developmental disorders: implications for brain function hillsdale. NJ: Lawrence Erlbaum.

Caplan, D. (2006). Aphasic deficits in syntactic processing. Cortex, 4216, 797-804.

Complex adaptive system. Wikipedia. https://en. wikipedia. org/wiki/Complex_adaptive_system.

(Accessed on February 8, 2020)

Corballis, M. (2002). From hand to mouth: the origins of language. Language Evolution, 98(1), 164-165.

Dabrowska, E. (2004). Language, mind and brain: some psychological and neurological experts constraints on theories of grammar. Edinburgh: Edinburgh University Press, 75.

Dorogovtsev, S. N. \& Mendes, J. F. F. (2002). Evolution of networks. Advances in Physics, 51(4), 1079-1145.

Enard, W., Przeworski, M., Fisher, S. E., Lai, C. S. L., Wiebe, V. \& Kitano, T., et al. (2002). Molecular evolution of FOXP2, a gene involved in speech and language. Nature, 418(6900), 869-872.

Gardner, B. T. \& Gardner. R. A. (1974). Comparing the early utterances of child and chimpanzee. In A. Pick (Ed.), Minnesota symposium on use of signs by chimpanzees with humans: child psychology. Minneapolis: University of Minnesota Press.

Gardner, H. (1974). The shattered mind. New York: Vintage.

Gibbs, R. W. \& Van, G. O. (2010). Adaptive cognition without massive modularity. Language and Cognition, 2(2), 149-176.

Gould, S. J. (1998). Leonardo's mountain of clams and the diet of worms: essays on natural history. New York: Harmony Books. 
Harris, N. G., Bellugi, U., Bates, E., Jones, W. \& Rossen, M. (1997). Contrasting profiles of language development in children with Williams and Down syndromes. Developmental Neuropsychology, 13(3), 345-370.

Hotting, K. , Rosier, F. \& Roder, B. (2004). Altered multisensory interaction in congenitally blind humans: an event-related potential study. Experimental Brain Research, 159(3), 370-381.

Jungé, J. A. \& Dennett, D. C. (2010). Multi-use and constraints from original use. Behavioral and Brain Science, 33(4), 277278.

Karmiloff, S. A. (1994). Beyond modularity: a developmental perspective on cognitive science. Cambridge, MA: MIT Press.

Karmiloff-Smith, A., Klima, E., Bellugi, U., Grant, J., \& Baron-Cohen, S. (1995). Is there a social module? language, face processing, and theory of mind in individuals with Williams syndrome. Journal of Cognitive Neuroscience, 7(2), $196-208$.

Karmiloff-Smith, A. (2001). Research into Williams syndrome: the state of the art. In Nelson, C. A. and Luciana, M. (eds.). Handbook of developmental cognitive neuroscience. Cambridge, MA: MIT Press.

Lai, C. S. L., Fisher, S. E., Hurst, J. A., Vargha-Khadem, F. \& Monaco, A. P. (2001). A fork-head gene is mutated in a severe speech and language disorder. Nature, 413(6685), 519-523.

Maess, B. S., Koelsch, T. C., Gunter \&Friederici, A. D. (2001). Musical syntax is processed in Broca's area: an MEG study. Nature Neuro-Science, 4(5), 540-545.

Machery, E. (2007). Massive modularity and brain evolution, Science, 74(5), 825-838, 827.

McGurk, H. \& MacDonald, J. (1976). Hearing lips and seeing voices. Nature, 264(5568), 746-748.

Mody, M., Studdert-Kennedy, M. \& Brady, S. (1997). Speech perception deficits in poor readers: auditory processing or phonological coding? Journal of Experimental Child Psychology, 64(2), 199-231.

Pinker, S. (1994). The language instinct. New York: William Morrow.

Pinker S. (1997). How the mind works. New York: W. W. Norton and Co.

Pinker, S. (2002). The blank slate. New York: Penguin.

Plaza, M., Gatignol, P., Leroy, M. \& Duffau, H. (2009). Speaking without Broca's area after tumor resection. Neurocase, 15 (4), 294-310.

Poeppel, D. (1996). A critical review of PET studies of phonological processing. Brain and Language, 55(3), 317-351.

Preston, L. (1999). Researchers find brain link between sight and touch. Emory Repor, November 8. Volume 52, No. 11. Retrieved from http: //www. emory. edu/EMORY_REPORT/erarchive/ 1999/November/ernovember. 8/11_8_99brain. html.

Prinz, J. J. (2002). Furnishing the mind: concepts and their perceptual basis. Cambridge, MA: MIT Press.

Prinz, J. (2006). Is the mind really modular? In R. J. Stainton (Ed.), Contemporary debates in cognitive science. Malden, MA: Blackwell Publishing.

Pulvermüller, F. (1999). Words in the brain's language. Behavioral and Brain Sciences, 22(2), 253-336.

Quartz, S. R. (2002). Toward a developmental evolutionary psychology: genes, development, and the evolution of the human cognitive architecture. In Scher S and Rauscher M (eds.), Evolutionary psychology: genes, development, and the evolution of the human cognitive architecture. alternative approaches. Dordrecht: Kluwer.

Smith, N. (1999). Chomsky: ideas and practices. Cambridge: Cambridge University Press.

Townsend, J., Wulfeck, B., Nichols, S. et al. (1995). Attentional deficits in children with developmental language disorder. Technical report CND-9513. San Diego: Center for Research in Language, University of California.

(Editor: Jasmine Liu ) 